\title{
The role of black hole spin and magnetic field threading the unstable neutrino disk in gamma ray bursts
}

\author{
A. Janiuk ${ }^{1}$ and Y.-F. Yuan ${ }^{2}$ \\ 1 Copernicus Astronomical Center, Polish Academy of Sciences, ul. Bartycka 18, 00-716 Warsaw, Poland \\ e-mail: agnes@camk.edu.pl \\ 2 Key Laboratory for Research in Galaxies and Cosmology, University of Science and Technology of China, \\ Chinese Academy of Sciences, Hefei 230026, PR China \\ e-mail: yfyuan@ustc.edu.cn
}

Received 19 June 2009 / Accepted 25 October 2009

ABSTRACT

\begin{abstract}
Aims. We report on the third phase of our study of the neutrino-cooled hyperaccreting torus around a black hole that powers the jet in gamma ray bursts. We focus on the influence of the black hole spin on the properties of the torus.

Methods. The structure of a stationary torus around the Kerr black hole is solved numerically. We take into account the detailed treatment of the microphysics in the nuclear equation of state that includes the neutrino trapping effect.

Results. We find that in the case of rapidly rotating black holes, the thermal instability discussed in our previous work is enhanced and is developed for much lower accretion rates. We also find the important role of the energy transfer from the rotating black hole to the torus via the magnetic coupling.
\end{abstract}

Key words. stars: gamma-ray bursts: general - black holes physics - accretion, accretion disks - neutrinos - instabilities magnetic fields

\section{Introduction}

Observed gamma ray bursts (GRBs) form at least two classes of events. Long GRBs $\left(T_{90}>2 \mathrm{~s}\right)$ are the the result of a collapsar in the core of an exploding massive star, as supported by the detections of their afterglows. Shorter bursts $\left(T_{90}<2 \mathrm{~s}\right)$ are likely to be due to the coalescence of a binary system containing two compact stars, as is observationally supported by the localization of their afterglows at the outskirts of their host galaxies (see e.g. the review of Zhang 2007). Moreover, it is possible that a third class of events exists, namely a fraction of the shortest bursts $\left(T_{90}<0.1 \mathrm{~s}\right)$, that can be produced via the evaporation of the primordial black holes (Cline et al. 2005).

Both the two main classes of bursts involve a stage of a newly formed black hole surrounded by an accreting torus, responsible for the production of a collimated jet. This jet is a source of a highly beamed emission observed in the gamma ray band. The accreting torus is either fed by the material from the stellar envelope, which partly falls back after the hypernova explosion or consumes the material from the disrupted neutron star debris. The torus accretion proceeds with highly hyper-Eddington rates on the order of a solar mass per second. Such hyper-accreting tori have been already discussed in a number of works (e.g. Popham et al. 1999; Di Matteo et al. 2002; Kohri et al. 2005; Chen \& Beloborodov 2007; Janiuk et al. 2004, 2007; Lei et al. 2009).

In the present paper we expand on our previous work, and we further investigate the properties and evolution of such hot and dense accretion tori. At the extreme densities and temperatures, determined by the hyper-Eddington accretion rate, the torus is cooled mainly by neutrino emission produced primarily by electron and positron capture on nucleons ( $\beta$ reactions). The model we develop here was first described in Janiuk et al. (2004) for the case of a non-rotating black hole and the nuclear matter with a simplified equation of state. We further developed this model in Janiuk et al. (2007), considering the more elaborate equation of state to account for the microphysics of the accreting plasma. In the latter work, we solved the equations for the disk structure and its time evolution by introducing the new EOS (equation of state) which includes the photodisintegration of helium, the condition of the $\beta$ equilibrium, and neutrino opacities. We selfconsistently calculated the chemical equilibrium in the gas consisting of helium, free protons, neutrons, and electron-positron pairs and computed the chemical potentials of the species as well as the electron fraction throughout the disk. We found an important property of our solutions: for very large accretion rates, the torus becomes viscously and thermally unstable in a narrow range of radii near the central black hole. The instability which results is an intrinsic property of the torus, when the helium is fully photodisintegrated in the neutrino opaque plasma.

In the present work, we further expand our model to account for the black hole rotation. The rotating black hole should naturally be produced in the center of a collapsar or after the merger event, when the collapsing material possesses a large amount of angular momentum. As a consequence, the black hole spin is a plausible mechanism that helps to launch the jets emitted in a narrow cone along the rotation axis. As shown in Janiuk et al. (2008), especially for the long duration GRBs the BH spin is required to sustain the central engine activity and the jet production for a sufficiently long time. Because these long GRBs often exhibit a very variable temporal structure (e.g. Beloborodov et al. 2000), it seems very important to study a possible instability mechanism that may account for the variability. We check here for which values of the black hole spin the instability may operate near the central black hole, depending on the accretion rate and viscosity in the disk. We also check what effects may be 
imposed on the torus and its unstable strip by the additional heating via the magnetic fields, threaded by the rotating black hole. The content of the article is as follows. First, we discuss the basic assumptions and present the main equations of the model. Second, we introduce the changes and corrections to the model that are the result of a black hole rotation with an arbitrary spin. Third, we introduce the energy extraction from the rotating black hole via the magnetic field, as an additional physical process that may operate in the gamma ray burst central engine. In Sect. 3 we present the results of our calculations, and in Sect. 4 we discuss the results and conclude.

\section{Model}

The model of a neutrino-cooled accreting torus was fully discussed in Janiuk et al. (2004, 2007). Here we briefly repeat the basic assumptions, and the model equations are given in Sect. 2.1. In Sect. 2.2 we describe the relativistic corrections to the model equations, which account for the black hole rotation in the Kerr metric. In Sect. 2.3 we discuss the additional heating term in the torus energy balance that comes from the rapid black hole rotation and magnetic dynamo.

\subsection{Basic equations}

The starting model for the torus evolution is calculated by solving the energy balance equation at a given accretion rate, $\dot{M}$, black hole mass, $M$, and viscosity, $\alpha$. The steady-state model is vertically averaged, with a surface density $\Sigma=H \rho$, and a height $H=c_{\mathrm{s}} / \Omega_{\mathrm{K}}$. Here the sound speed is $c_{\mathrm{s}}=\sqrt{P / \rho}, P$ is the total pressure and $\Omega_{\mathrm{K}}=\sqrt{G M / r^{3}}$ is the Keplerian angular velocity. The viscosity is described as in Shakura \& Sunyaev (1973), with $\tau_{r \varphi}=-\alpha P$, and $0<\alpha<1$ is a model parameter.

Throughout the calculations we adopt a black hole mass of $M=3 M_{\odot}$ and several chosen values of $\alpha$ and $\dot{M}$.

For the equation of state, we assume that the torus consists of helium, electron-positron pairs, free neutrons and protons. The total pressure is due to the free nuclei and pairs, helium, radiation and the trapped neutrinos:

$P=P_{\text {nucl }}+P_{\mathrm{He}}+P_{\mathrm{rad}}+P_{v}$

where $P_{\text {nucl }}$ includes free neutrons, protons, and the electronpositron pair gas in beta equilibrium, taking into account the partial degeneracy and relativistic temperatures of the species (Yuan 2005; Yuan \& Heyl 2005). The helium is generally nonrelativistic and non-degenerate; therefore, its pressure is given by the ideal gas EOS. Its number density is calculated using the formula of Popham et al. (1999)

$X_{\text {nuc }}=295.5 \rho_{10}^{-3 / 4} T_{11}^{9 / 8} \exp \left(-0.8209 / T_{11}\right)$.

The radiation pressure includes the factor due to the electronpositron pairs, and the neutrino pressure is calculated for the trapped neutrinos, using the two-stream approximation (Popham \& Narayan 1995; Di Matteo et al. 2002). We consider both the neutrino transparent and opaque regions as well as the transition between the two, and in order to determine the distribution function of the partially trapped neutrinos we use a "gray body" model (Sawyer 2003), consistent with the two-stream approximation.

The chemical potentials, or equivalently the ratio of free protons, $x=n_{\mathrm{p}} / n_{\mathrm{b}}$, are determined from the condition of equilibrium between the transition reactions from neutrons to protons and from protons to neutrons for a given baryon number density, $n_{\mathrm{b}}$, and temperature, $T$. These reactions are electron and positron capture on nucleons and neutron decay (see Kohri et al. 2005; Janiuk et al. 2007). The closing equations for the EOS are the conservation of the baryon number, $n_{\mathrm{n}}+n_{\mathrm{p}}=n_{\mathrm{b}} X_{\mathrm{nuc}}$, and the charge neutrality (Yuan 2005).

For the neutrino cooling of the torus, we consider the electron-positron pair annihilation, bremsstrahlung, plasmon decay and beta reactions. Each of these emission processes has a reverse one, which leads to neutrino absorption, and we calculate the absorptive optical depth $\tau_{\mathrm{a}, v_{i}}$ for the neutrinos of the three flavors. In addition, the free escape of neutrinos from the disc is limited by scattering, and we calculate the scattering optical depth $\tau_{\mathrm{s}}$. The neutrino cooling rate is therefore in the neutrinothick torus given by

$Q_{v}^{-}=\frac{\frac{7}{8} \sigma T^{4}}{\frac{3}{4}} \sum_{i=e, \mu} \frac{1}{\frac{\tau_{\mathrm{a}, v_{i}}+\tau_{\mathrm{s}}}{2}+\frac{1}{\sqrt{3}}+\frac{1}{3 \tau_{\mathrm{a}, v_{i}}}}$.

Apart from the neutrino emission, the disk is also cooled by advection, radiation and photodissociation of helium nuclei. The advective cooling is determined approximately as:

$Q_{\mathrm{adv}}^{-}=\Sigma v_{r} T \frac{\mathrm{d} S}{\mathrm{~d} r}=q_{\mathrm{adv}} \frac{\alpha P H T}{\Omega \rho r^{2}} S$,

where $q_{\mathrm{adv}} \propto \mathrm{d} \ln S / \mathrm{d} \ln r \propto\left(\mathrm{d} \ln T / \mathrm{d} \ln r-\left(\Gamma_{3}-1\right) \mathrm{d} \ln \rho / \mathrm{d} \ln r\right) \approx$ 1.0. The entropy density $S$, similarly to the total pressure, is the sum of four components:

$S=S_{\text {nucl }}+S_{\mathrm{He}}+S_{\text {rad }}+S_{v}$

and we calculate the entropy from the EOS.

The radiative cooling is

$Q_{\mathrm{rad}}^{-}=\frac{3 P_{\mathrm{rad}} c}{4 \tau}=\frac{11 \sigma T^{4}}{4 \kappa \Sigma}$

where we adopt the Rosseland-mean opacity $\kappa=0.4+0.64 \times$ $10^{23} \rho T^{-3}\left[\mathrm{~cm}^{2} \mathrm{~g}^{-1}\right]$, and the coefficient $11 / 4$ accounts for the contribution of electron-positron pairs.

The cooling rate due to the photodisintegration of $\alpha$ particles is:

$Q_{\text {photo }}^{-}=6.28 \times 10^{28} \rho_{10} v_{r} \frac{\mathrm{d} X_{\text {nuc }}}{\mathrm{d} r} H$

and $X_{\text {nuc }}$ is given by Eq. (2).

Finally, the hydrodynamic equations we use to calculate the disk structure are the standard mass, energy and momentum conservation. The viscous heating rate is determined by the shear, $\tau_{\mathrm{r} \varphi}$ and can be written in terms of $\alpha$ :

$Q_{\mathrm{visc}}^{+}=\frac{3}{2} \alpha \Omega H P$.

The total flux of energy generated at the radius $r$ is determined by the global model parameters, i.e. the black hole mass and accretion rate:

$F_{\text {tot }}=\frac{3 G M \dot{M}}{8 \pi r^{3}} f(r)$

where $f(r)$ stands for the inner boundary condition and will be given below. In order to calculate the initial stationary configuration, we solve the energy balance:

$F_{\text {tot }}=Q_{\mathrm{visc}}^{+}=Q_{\mathrm{adv}}^{-}+Q_{\mathrm{rad}}^{-}+Q_{v}^{-}+Q_{\mathrm{photo}}$. 
The above equations are appropriate in the Schwarzschild black hole case, studied in our previous work (Janiuk et al. 2004, 2007), and must be modified in the Kerr metric. In Sect. 2.2, we include the appropriate correction factors to the disk structure equations to introduce the Kerr metric and account for the black hole rotation.

\subsubsection{Instability in the disk}

The stability analysis of the neutrino-ccoled disk in the $\beta$-equlibrium was presented in Janiuk et al. (2007). The criterion for a viscously unstable disk is:

$$
\frac{\partial \dot{M}}{\partial \Sigma}<0
$$

and means that, at a given radius, the underdense (overdense) region evolves slower (faster), which results in material being evacuated from some rings of the disk, while being piled up in the others. The unstable region can be conveniently located in the steady-state solutions, on the surface density - accretion rate (or temperature) plane. On this plane, the branch of thermal equilibrium solutions, which has a negative slope, is unstable to the surface density perturbations. This branch is also thermally unstable, and we have

$\frac{\partial \log Q^{+}}{\partial \log T}>\frac{\partial \log Q^{-}}{\partial \log T}$

and therefore any small increase (decrease) in temperature leads to more (less) heating rate with respect to the cooling.

We found that for a non-rotating black hole, the neutrinocooled disk was generally stable for all radii if $\dot{M} \leq 10 \mathrm{M}_{\odot} \mathrm{s}^{-1}$, while at larger accretion rates the unstable region appeared at the innermost radii. The instability grows because the energy balance changes which in turn is caused by the photodisintegration of helium, while the neutrinos are trapped in the opaque plasma. In the unstable region, the electrons and protons become nondegenerate, and the electron fraction rises inwards with radius. The changes in the electron fraction influence the total pressure and the energy dissipation rate, so that the heating and cooling balance is affected and the local accretion rate increases. We found that if we used the highest accretion rates $\left(12 M_{\odot} \mathrm{s}^{-1}\right)$ and the non-rotating black hole, the radial extension of the unstable part of the disk was up to $\sim 8$ Schwarzschild radii.

\subsection{Black hole rotation}

The structure of the relativistic accretion disk is calculated using the correction factors derived in Riffert \& Herold (1995) for a Kerr black hole (see e.g. Reynoso et al. 2006). The black hole spin is parameterized by a dimensionless specific angular momentum (Bardeen 1970), $a=c J / G M^{2}$, and the following functions of $a, M$, and radial coordinate $r$ are introduced:

$$
\begin{aligned}
& A=1-\frac{2 G M}{c^{2} r}+\left(\frac{G M a}{c^{2} r}\right)^{2} \\
& B=1-\frac{3 G M}{c^{2} r}+2 a\left(\frac{G M}{c^{2} r}\right)^{3 / 2} \\
& C=1-4 a\left(\frac{G M}{c^{2} r}\right)^{3 / 2}+3\left(\frac{G M a}{c^{2} r}\right)^{2} \\
& D=\frac{1}{2 \sqrt{r}} \int_{r_{\mathrm{ms}}}^{r} \frac{\frac{x^{2} c^{4}}{G^{2}}-6 \frac{M x c^{2}}{G}+8 a \sqrt{\frac{M^{3} x c^{2}}{G}}-3 a^{2} M^{2}}{\sqrt{x}\left(\frac{x^{2} c^{4}}{G^{2}}-3 \frac{M x c^{2}}{G}+2 a \sqrt{\frac{M^{3} x c^{2}}{G}}\right)} \mathrm{d} x .
\end{aligned}
$$

The above coefficients approach unity at large radii and for small spin parameters. The inner boundary of the disk is located at the last stable circular orbit, depending on the black hole spin (Bardeen et al. 1972):

$r_{\mathrm{ms}}=\frac{G M}{c^{2}}\left(3+z_{2} \pm \sqrt{\left(3-z_{1}\right)\left(3+z_{1}+2 z_{2}\right)}\right)$

where $z_{1}=1+\left(1-a^{2}\right)^{1 / 3}\left((1+a)^{1 / 3}+(1-a)^{1 / 3}\right), z_{2}=\left(3 a^{2}+z_{1}^{2}\right)^{1 / 2}$, and the upper sign in Eq. (17) is for a retrograde orbit, while the bottom sign is for a direct orbit around a black hole. (In this work we always consider the direct orbits.)

The increase of gravity in the close vicinity of the rotating black hole leads in consequence to a smaller disk height, which will be given by the hydrostatic equilibrium:

$H=\sqrt{\frac{P}{\rho}} \frac{1}{\Omega} \sqrt{\frac{B}{C}}$.

The angular velocity in the disk around a spinning black hole is given by:

$\Omega=\frac{c^{3}}{G M\left(\left(\frac{r c^{2}}{G M}\right)^{3 / 2}+a\right)}$

The viscous shear will be modified as:

$\tau_{r \varphi}=-\alpha P \frac{A}{\sqrt{B C}}$.

Consequently, the heating and cooling terms in the energy balance equation will be affected by the above correction factors. Also, the equation for the energy flux generated in the disk, Eq. (9), will now be specified in the Kerr metric. Therefore, instead of the Newtonian boundary condition, we use the function:

$f(r)=\frac{D}{B}$.

This factor is equal to zero at the $r_{\mathrm{ms}}$ radius and approaches unity at large radii. This asymptotic behavior of $f(r)$ is the same as for the boundary condition derived in Chen \& Beloborodov (2007), who used more complex formalism in the Kerr metric. The torque imposed on the disk due to magnetic coupling with the black hole (see below) does not change the no-torque inner boundary condition either.

\subsection{Magnetic heating}

The spinning black hole may transfer its rotation energy to the jet via the Blandford-Znajek (BZ) process (Blandford \& Znajek 1977) as well as to the accretion disk, via the magnetic coupling (Li \& Paczyński 2000). In the latter, the closed lines of the magnetic field connect the black hole horizon with the disk, and if the $\mathrm{BH}$ rotates faster than the surrounding disk, the torque is exerted and the energy and angular momentum are extracted from the $\mathrm{BH}$. On the other hand, the direction of the energy transfer may be reversed if the disk angular velocity is larger than that of the black hole.

The details of the process depend on the structure of the magnetic field. Here we follow the assumptions of Wang et al. (2003a,b) that the magnetic field is constant at the horizon and varies as a power law with the disk radius. We also use the mapping relation between the angular coordinate, $\theta$, on the $\mathrm{BH}$ horizon and the disk radius, $r$, derived by these authors. Due to this 
relation, the closed magnetic filed lines are attached not only to the inner edge of the disk, but connect the horizon region of smaller $\theta$ with outer disk radii up to some maximum radius $r_{\text {out }}$. Therefore, the disk inner ring, between $r_{\mathrm{ms}}$ and $r_{\text {out }}$, will be affected by the energy transfer to and from the rotating black hole, depending on its spin and magnetic field configuration. Because the inner regions of the disk are also subject to the thermal instability, we intend to check what effect on this instability may arise due to the energy transfer between the black hole and the disk.

The mapping relation is defined by the following change of variables (Wang et al. 2003a,b):

$\sin \theta d \theta=-G(a, \xi, n) \mathrm{d} \xi$

and

$\cos \theta=\int_{1}^{\xi} G(a, \xi, n) \mathrm{d} \xi$

where

$$
\begin{aligned}
& G(a, \xi, n)= \\
& -\frac{\xi^{1-n} \chi_{\mathrm{ms}}^{2} \sqrt{1+a^{2} \chi_{\mathrm{ms}}^{-4} \xi^{-2}+2 a^{2} \chi_{\mathrm{ms}}^{-6} \xi^{-3}}}{2 \sqrt{\left(1+a^{2} \chi_{\mathrm{ms}}^{-4}+2 a^{2} \chi_{\mathrm{ms}}^{-6}\right)\left(1-2 \chi_{\mathrm{ms}}^{-2} \xi^{-1}+a^{2} \chi_{\mathrm{ms}}^{-4} \xi^{-2}\right)}} .
\end{aligned}
$$

Here the dimensionless radius is $\xi=r / r_{\mathrm{ms}}, \chi=\sqrt{r / r_{\mathrm{g}}}$ and $r_{\mathrm{g}}=G M / c^{2}$. The mapping function $G(a, \xi, n)$ depends on the black hole spin, which is a parameter of our model, and on the strength of the magnetic field at a given radius. The magnetic field is assumed to vary as (Blandford 1977):

$B_{\mathrm{Z}} \propto \xi^{-n}$

and the index $n$ is a model parameter.

The magnetic coupling between the rotating black hole and the disk exerts a torque, which equals to:

$T_{\mathrm{MC}}=T_{0} 4 a\left(1+\sqrt{1-a^{2}}\right) \int_{0}^{\pi / 2} \frac{(1-\beta) \sin ^{3} \theta \mathrm{d} \theta}{2-\left(1-\sqrt{1-a^{2}}\right) \sin ^{2} \theta}$,

where $\beta=\Omega / \Omega_{\mathrm{H}}$ is the ratio between the angular velocity in the disk, given by Eq. (19) and the velocity at the black hole horizon is given by

$\Omega_{\mathrm{H}}=a /\left(2 r_{\mathrm{g}}\left(1+\sqrt{1-a^{2}}\right)\right)$.

The integration over the coordinate $\theta$ from $\theta_{\min }$ to $\theta_{\max }$ is equivalent to the integration over the disk radius from $\xi_{\max }$ to $\xi_{\text {min }}$. Here we set $\xi_{\min }=\xi_{\mathrm{ms}}=1$ at $\theta=\pi / 2$ and for simplicity we assume that no open magnetic field lines take the black hole rotation power to infinity, i.e. the BZ effect is vanishing and $\xi_{\max }$ has its largest value for $\theta_{\min }=0$.

The normalization of the torque is (Wang et al. 2003a,b):

$T_{0}=3.26 \times 10^{45}\left(\frac{B_{\mathrm{H}}}{10^{15} G}\right)^{2}\left(\frac{M}{M_{\odot}}\right)^{3} \mathrm{~g} \mathrm{~cm}^{2} \mathrm{~s}^{-2}$

and the energy density of the magnetic field is proportional to the total pressure in the disk (Moderski et al. 1998):

$B_{\mathrm{H}}^{2} \propto 8 \pi P^{\max }$.

In general, the proportionality coefficient in the above equation can be on the order of 1.0, however, some departures from the

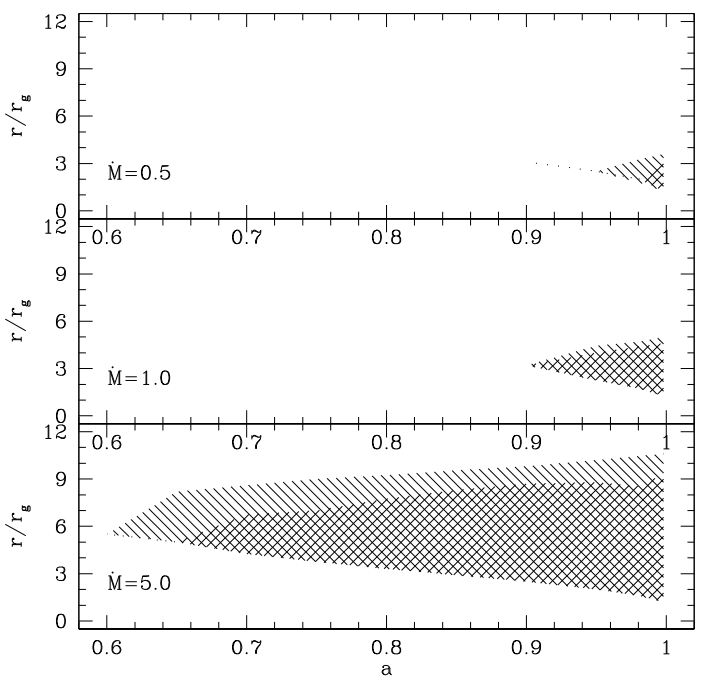

Fig. 1. The parameter space for which the unstable solution is found, in the plane radius - spin parameter. The bottom panel shows the results for the accretion rate $\dot{M}=5.0 M_{\odot} \mathrm{s}^{-1}$, the middle panel is for $\dot{M}=$ $1.0 M_{\odot} \mathrm{s}^{-1}$ and the top panel is for $\dot{M}=0.5 M_{\odot} \mathrm{s}^{-1}$. The two values of viscosity were used: $\alpha=0.1$ (shaded area) and $\alpha=0.3$ (cross-shaded area).

equipartition of magnetic energy are possible. In case of the $\alpha$ disks, the ratio of $B_{\mathrm{H}}^{2} / 8 \pi P^{\mathrm{max}}=\beta_{\mathrm{mag}}$ cannot be too high and will rather be on the order of $\alpha$ (Moderski, private communication).

Finally, the energy transfer from the rotating black hole to the accretion disk results in the additional heating term:

$Q_{\mathrm{MC}}^{+}=\frac{T_{\mathrm{MC}} \Omega}{4 \pi r^{2}}$

\section{Results}

\subsection{Disk instability for a rotating black hole}

We found that the thermal instability of the neutrino cooled disk, previously studied for the case of Schwarzschild black holes in Janiuk et al. (2007), is also present in the disks of Kerr black holes. The instability arises in the inner parts of the disk, where the helium is fully photodisintegrated, the nuclei pressure is locally lower, while the neutrino opacity is large and the dominant pressure terms are due to the neutrinos and radiation. In our previous work, we found for a non-rotating black hole that the radial extension of the unstable strip was up to about 8 Schwarzschild radii. The accretion rate for which the instability appeared had to be extremely large, on the order of 12 solar masses per second. The instability was manifested in both stationary models, as a phase transition to a distinct branch of distribution for various quantities (temperature, density, electron fraction, chemical potentials of species, etc.) as well as in the time dependent evolution of the disk (variability in time).

In this work, we calculated a grid of stationary models in the Kerr metric to check for the location of the unstable strip depending on the black hole spin. We used moderate accretion rates and several viscosity parameters. In Fig. 1 we show the radial extension of the unstable strip as a function of the spin parameter $a$, for three values of the accretion rate: $\dot{M}=0.5,1.0$ and 5.0 $M_{\odot} \mathrm{s}^{-1}$. We find that for the extreme Kerr black hole, the instability appears already at very low accretion rates of 0.5 solar masses per second. The dependence on $\alpha$ is rather weak. In general, the larger the viscosity, the larger the $\mathrm{BH}$ spin needed for the thermal instability to appear at a given accretion rate. Also, 


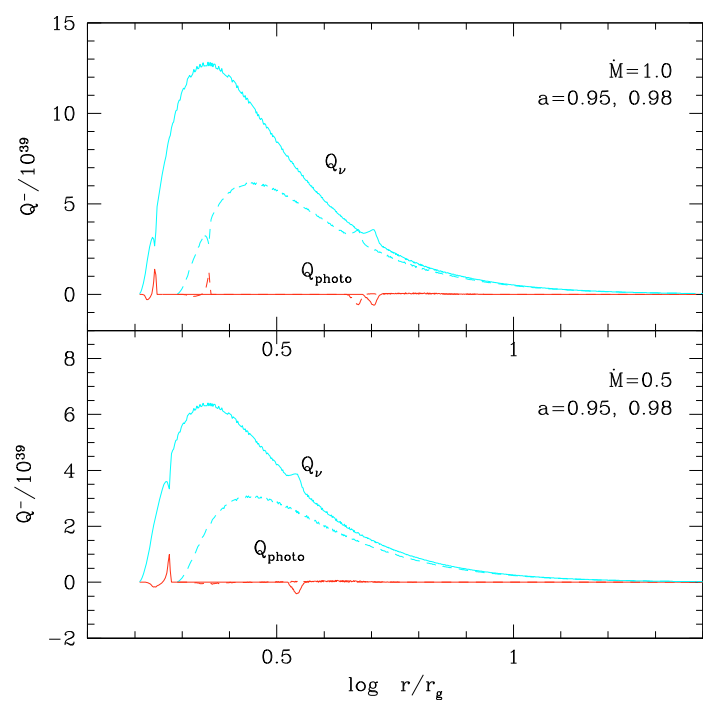

Fig. 2. The main cooling terms in the disk as a function of radius: neutrino cooling (thick blue lines) and helium photodisintegration (thin red lines). The bottom panel shows the results for the accretion rate $\dot{M}=0.5 M_{\odot} \mathrm{s}^{-1}$ and the upper panel is for $\dot{M}=1.0 M_{\odot} \mathrm{s}^{-1}$. The two values of the black hole spin parameter are shown: $a=0.98$ (solid lines) and $a=0.95$ (dashed lines). The viscosity parameter is $\alpha=0.1$.

for larger alpha, the instability strip is narrower. As shown in the upper panel of the figure, only for $a>0.95$ at a viscosity $\alpha=0.1$, and for $a>0.98$ at $\alpha=0.3$ the unstable solutions are found at this low accretion rate. For lower accretion rates, we checked $\dot{M}=0.1 \dot{M}_{\odot} \mathrm{s}^{-1}$, and no unstable solutions were found down to $\alpha=0.03$ and at $a=0.998$.

In general, the predicted trend is conserved: the higher the accretion rate and the larger the black hole spin, the larger the extension of the unstable strip. The trend with viscosity is the following: for larger viscosity parameters the extension of the unstable zone shrinks.

In Fig. 2 we show the dominant cooling terms as a function of radius. The terms are due to the neutrino emission, $Q_{v}$, and helium photodisintegration, $Q_{\text {photo }}$. The other cooling terms, due to radiation and the advection of energy, are incorporated in our model, but they are a few orders of magnitude smaller than the neutrino cooling. Therefore we do not show these terms on the plot. The $Q_{\text {photo }}$ term at large radii can have a negative value locally, which means that the helium nuclei are created at this radius.

The unstable strip in the accretion disk can be localized between the two spikes in the $Q_{\text {photo }}$ distribution. The first spike is very close to the inner edge of the accretion disk with a small offset because of the inner boundary condition (cf. Eq. (21)). Due to this condition, no torque is induced on the inner edge. This results in the zero viscous dissipation rate, and the plasma temperature drops close to the inner edge (in practice, for numerical reasons we set our inner radius of the computational domain slightly above inner edge, to avoid singularities). Note that the torque induced by the magnetic coupling between the disk and the black hole is transported only outwards with the radius, while at the inner edge of the disk the torque is still zero. We also note that the density drop at the inner edge is compensated for by a large radial velocity, because we take into account the advective cooling. Therefore, despite the small disk thickness, the accretion rate is kept constant. Above the inner edge of the torus, the temperature reaches $10^{10}-10^{11} \mathrm{~K}$, while the density exceeds $10^{9} \mathrm{~g} \mathrm{~cm}^{-3}$, and the helium nuclei can appear. The plasma

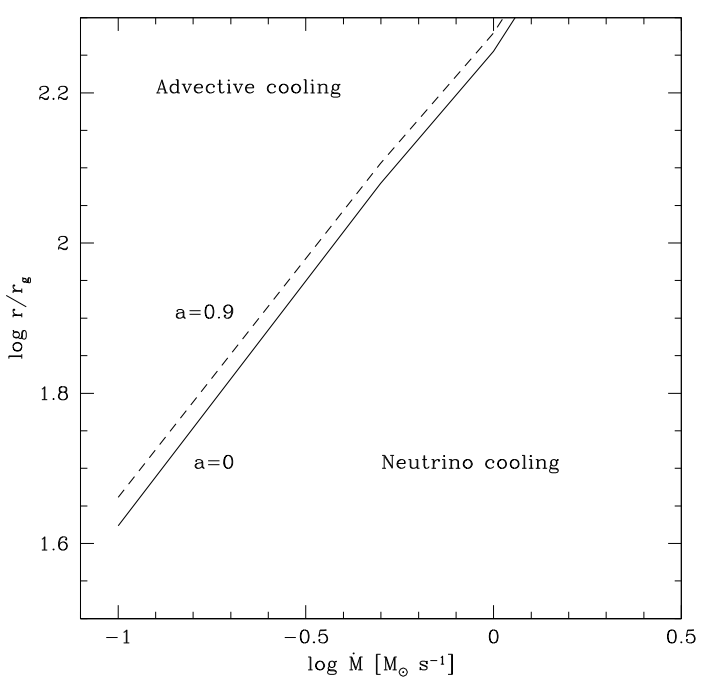

Fig. 3. The parameter space for which the disk is dominated by the neutrino cooling (NDAF). We plot the maximum radius of NDAF (in the units of $r_{\mathrm{g}}=G M / c^{2}$ ) as a function of accretion rate, for two chosen values of black hole spin: $a=0$ (solid line) and $a=0.9$ (dashed line).

consists of free neutrons, protons and electron-positron pairs, and if the photon flux is sufficient, the helium nuclei are photodisintegrated. Depending on the model parameters, the outer radius of this strip can reach up to 12 gravitational radii (Fig. 1). Outwards of this radius, the helium is again synthesized, and its photodisintegration contributes to the energy balance in the outer disk (with some small fluctuations in $Q_{\text {photo }}$ up to $r_{\text {out }}$, not visible in the figure).

The neutrino cooling term is always a dominant one in all the models. The larger the accretion rate and the black hole spin, the hotter is the disk, and hence the neutrino cooling is larger. For the rotating black hole, the inner disk radius shifts closer (cf. Eq. (17)), and the maximum in the heating and cooling distribution is shifted inwards as well.

We note that our model works well in the neutrino-cooling dominated region, which is always inside the radius $r_{\max }$. This "neutrino ignition radius" strongly depends on the accretion rate and weakly depends on the black hole spin. Outside this region, the flow is dominated by advection, and neutrino emission is inefficient in cooling the disk (see also Chen \& Beloborodov 2007, note however that we do not consider the gravitational instability, which according to these authors is present at large radii and large accretion rates, nor do we study the case of the smallest accretion rates, for which the neutrino cooling is inefficient throughout the disk at all radii). In Fig. 3 we indicate the parameter space for which the neutrino cooling is dominant. The instability we discuss here, as well as the magnetically coupled region we discuss below, are both located well inside the neutrino cooled region.

\subsection{Magnetic coupling between the disk and rotating black hole}

The mapping relation between the polar coordinate on the black hole horizon and the disk radius implies that there exists a maximum radius, defining the region of influence of the rotating black hole. Below this radius, at $\xi<\xi_{\text {out }}$, the energy may be transferred to and/or from the black hole to the disk. Above this radius, there are no closed magnetic field lines, and the disk is no longer subject to the heating by the black hole energy extraction. 


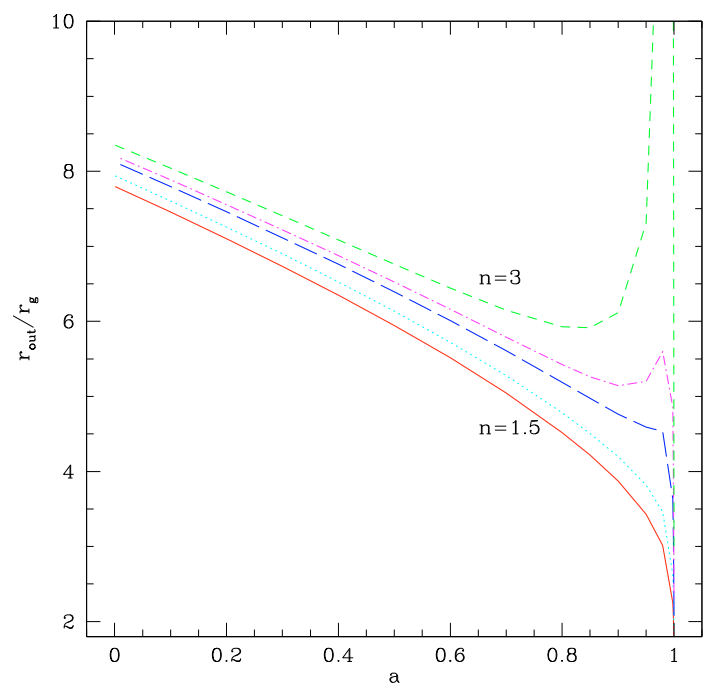

Fig. 4. The radial extension of the region threaded by magnetic coupling between the disk and rotating black hole as a function of its spin. The solid line marks the power law index of magnetic field $n=1.5$, the dotted line is for $n=2$, the long-dashed line is for $n=2.5$, the dotteddashed line is for $n=2.7$ and the short dashed line is for $n=3$.

In Fig. 4 we show the dependence of the radial extension of the magnetically coupled region on the black hole spin and the magnetic field distribution. The latter is parameterized by the index $n$ in Eq. (25), and the figure shows several examples for $n=1.5,2.0,3.0$ and 6.0. As was found by Wang et al. (2003a,b), the influence of the black hole rotation is concentrated in the closest vicinity of the inner disk edge and the radius $\xi_{\text {out }}=r_{\text {out }} / r_{\mathrm{ms}}$ is very small for small values of index $n$. For $n \leq 2.0$ the largest $\xi_{\text {out }}$ is about 2.2, at the black hole spin $a=0.98$, and then it drops. The result for larger $n$ are quantitatively the same as in Wang et al. (2003a,b), and they differ only quantitatively. For $n=3$ the maximum is $\xi_{\text {out }}>10.0$ at $a=0.998$, while those authors had a value about $\xi_{\text {out }} \approx 4.0$, which in turn we obtained for $n=2.7$. The reason is probably the sensitivity of the results to the numerical method used to compute the integrals in Eq. (26). For $n>3.0$ and large $a$, the size of the region $\xi_{\text {out }}$ goes to infinity, and the larger $n$, the smaller is the critical spin parameter at which that happens (see Wang et al. 2002).

Note that actually in Fig. 4 we plot the results in the units of the gravitational radius, $r_{\mathrm{g}}$, instead of $r_{\mathrm{ms}}$ radius, i.e. the dependence on the black hole spin is accounted for also in the $r_{\mathrm{ms}}$ value. Therefore we can directly compare the extension of the magnetically coupled region with the extension of the thermal instability in the disk, presented in Fig. 1. In these units, the size of the magnetically coupled region decreases with $a$ because the $r_{\mathrm{ms}}$ radius is also smaller (the inner edge is closer to the black hole) if the spin is large. For the most interesting cases of the large spin, the outer radius of magnetically coupled region is usually well inside the thermal instability strip, provided the accretion rate is sufficient for the thermal instability to occur and $n$ is rather small. However, if $n \geq 3$, the magnetically coupled region may extend to larger radii than the thermally unstable strip, especially for moderately small accretion rates.

However, even if the size of the magnetically threaded strip may be small, the energy input given to the disk by a rotating black hole is quite large. In Fig. 5 we show the maximum magnetic torque induced in the disk by the black hole rotation, depending on its spin, for several values of the index $n$. The value

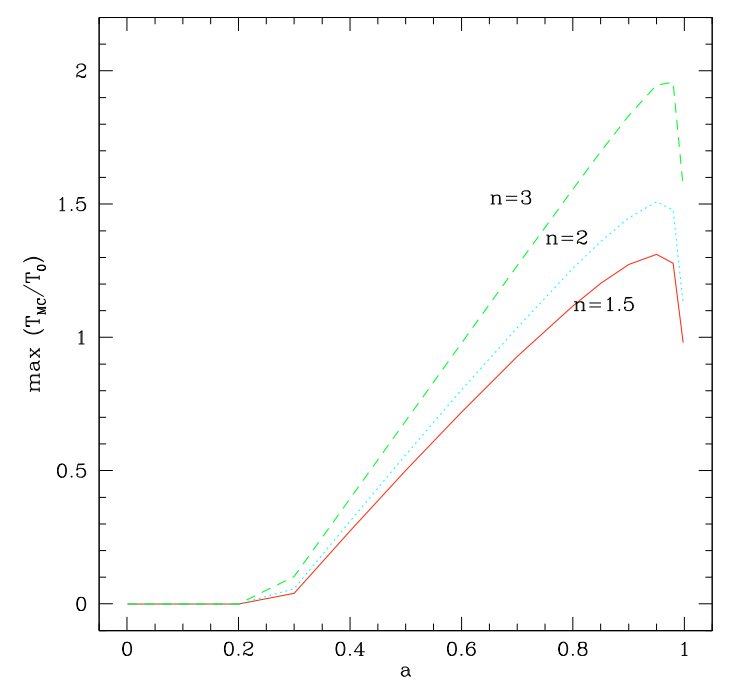

Fig. 5. The maximum magnetic torque induced in the disk, as a function of the black hole spin. The solid line marks the power law index of magnetic field $n=1.5$, the dotted line is for $n=2$, the long dashed line is for $n=2.5$, the dotted-dashed line is for $n=2.7$ and the short dashed line is for $n=3$.

of the torque increases with the radius and saturates at $\xi_{\text {out }}$ at its maximum value. This value is correlated to both index $n$ and spin, decreasing only for the extreme Kerr black holes. The value of the maximum $T_{\mathrm{MC}}=0$, obtained for the most slowly rotating black holes, means that in fact for these parameters the torque is negative throughout the magnetically coupled region. This means that the rotation energy is transferred only from the disk to the black hole. On the other hand, for the fast rotating black holes and large $n$, the value of $T_{\mathrm{MC}}$ may be large and positive at the outer parts of the magnetically coupled region while negative in the innermost radii. The reason for this is that if $r_{\mathrm{ms}}$ is sufficiently small, the innermost disk parts rotate faster than the black hole, and the energy is extracted from the inner disk to spin up the black hole.

Now, we study how the magnetic coupling between the disk and the rotating black hole will influence the thermal-viscous instability, found by Janiuk et al. (2007). This thermal instability was studied in the case of the non-rotating black hole and found to require very high accretion rates. In the present work we confirmed that the instability occurs also for the rotating black holes and needs smaller accretion rates (Sect. 3.1). To further investigate the role of the black hole rotation, we calculated a grid of models parameterized by the accretion rate $\dot{M}$, the black hole spin $a$ as well as the magnetic field power law index $n$ and its normalization $\beta_{\mathrm{mag}}$, which describe the strength of the magnetic coupling.

In Fig. 6 we show the density profiles for two values of the accretion rate $\dot{M}=0.5$ and $1.0 M_{\odot} \mathrm{s}^{-1}$, and several spin parameters in the range from $a=0.9$ to 0.998 . We concentrate on the innermost region of the accretion disk, where both the thermalviscous instability and the magnetic coupling overlap. Therefore the figures show the region up to the radius $r=15 r_{\mathrm{ms}}$. As discussed in Janiuk et al. (2007), the thermal-viscous instability results in a local density decrease accompanied by the temperature rise in the inner strip of the disk. Outwards of that strip, a stable solution is found, for which the density and temperature smoothly decrease with radius.

Now we compare our previous unstable solutions, calculated under the assumption of no magnetic torque (i.e. $\beta_{\mathrm{mag}}=0$, shown by the thinner lines), with the new solutions with the 
A. Janiuk and Y.-F. Yuan: Unstable GRB disk around a spinning black hole

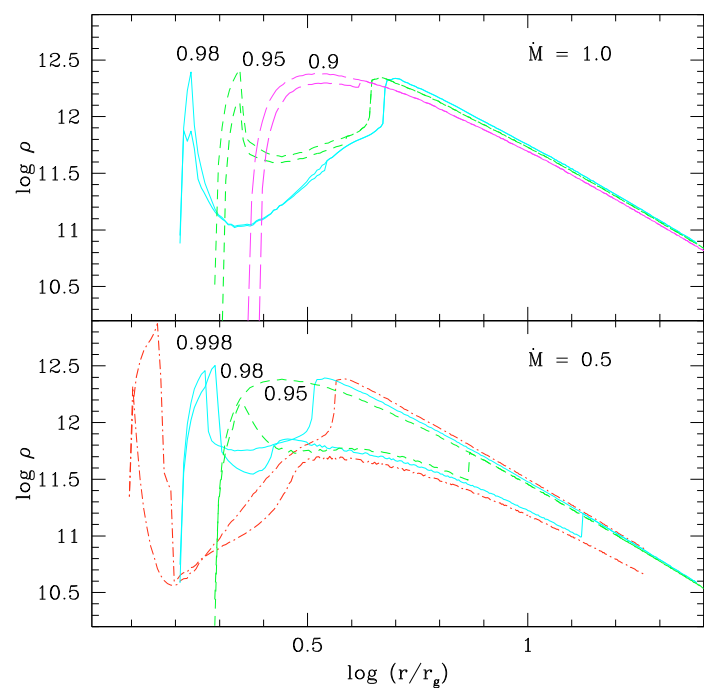

Fig. 6. The density profiles for the magnetically heated/cooled disks (thick lines), for several values of the black hole spin: $a=0.998$ (dotdashed lines), $a=0.98$ (solid lines), $a=0.95$ (short dashed lines) and $a=0.9$ (long dashed lines). The thinner lines show the case when the magnetic torque was neglected. The parameters were $\dot{M}=1.0 M_{\odot} \mathrm{s}^{-1}$ (top panel) and $\dot{M}=0.5 M_{\odot} \mathrm{s}^{-1}$ (bottom panel). The magnetic torque parameters were $n=2, \beta_{\mathrm{mag}}=0.1$ (top panel), and $n=3, \beta_{\mathrm{mag}}=0.5$ (bottom panel). The viscosity parameter is $\alpha=0.1$.

magnetic torque included (i.e. $\beta_{\text {mag }}=0.5$ or 0.1 , shown by the thicker lines). We note that the density dip is deeper whenever the energy is transferred from the black hole to the disk. Only very close to the inner edge of the disk, for large $\mathrm{BH}$ spins and an index $n \sim 3$, we have the black hole extracting energy from the disk. When this is coupled to the thermal instability, the density at the innermost radii increases, and the instability strip effectively shifts towards larger radii. We can identify the outer edge of the instability strip with the local maximum of the density. Therefore, this point is shifted towards a smaller radius, and in turn the whole unstable strip is narrower. However, if both $a$ and $n$ are sufficiently large, the magnetically coupled region extends well outside the instability strip (cf. Fig. 6; bottom panel). In this case, the density profile is still affected, and the density is systematically lower than in the respective model without the magnetic torque. However, now the density also decreases smoothly with the radius, and we do not find this region unstable.

For a smaller index of $n=2$, the magnetically coupled region does not cover the whole instability strip. This is shown in the top panel of Fig. 6. Therefore we have the density decrease only in some part of that strip, while outwards the solution matches the $Q_{\mathrm{MC}}{ }^{-}=0$ case. The effect is smaller in these models than in case of $n=3$, because we now use the $\beta_{\text {mag }}=0.1$ instead of $\beta_{\text {mag }}=0.5$, so that the magnetic heating does not exceed the total emitted flux $F_{\text {tot }}$. For $n=2$ and $a<0.98$, the magnetic torque is positive everywhere, i.e. there is no region where the energy is extracted from the disk towards the black hole, and there is no density rise on the inner edge.

The local density dip is also produced by the magnetic term in these models where there is no thermal instability. This is shown in Fig. 6 for $\dot{M}=0.5, a=0.95$ (bottom panel) and for $\dot{M}=1.0, a=0.9$ (upper panel). The radial extension, depth and localization of this dip are determined by the parameters $n$, $a$ and $\beta_{\mathrm{mag}}$.

In Fig. 7 we plot the temperature profiles for the same models as those shown in Fig. 6. As we mentioned above, the local

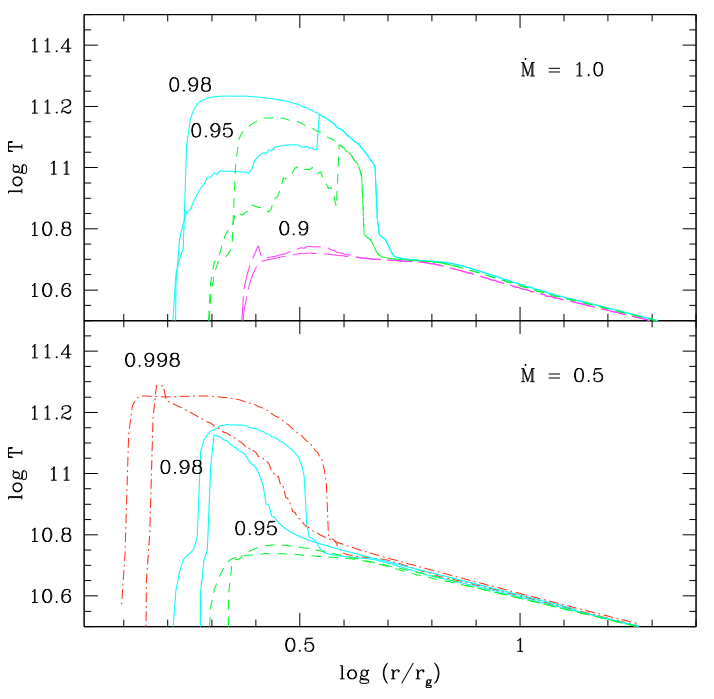

Fig. 7. The temperature profiles for the magnetically heated/cooled disks (thick lines), for several values of the black hole spin: $a=0.998$ (dot-dashed lines), $a=0.98$ (solid lines), $a=0.95$ (short dashed lines) and $a=0.9$ (long dashed lines). The thinner lines show the case when the magnetic torque was neglected. The parameters were $\dot{M}=1.0 M_{\odot} \mathrm{s}^{-1}$ (top panel) and $\dot{M}=0.5 M_{\odot} \mathrm{s}^{-1}$ (bottom panel). The magnetic torque parameters were $n=2, \beta_{\mathrm{mag}}=0.1$ ( top panel), and $n=3, \beta_{\text {mag }}=0.5$ (bottom panel). The viscosity parameter is $\alpha=0.1$.

density dip is accompanied by the temperature excess in the unstable region. However, when that region is magnetically threaded by a rotating black hole, the excess is smaller and extends to a smaller radius than for $Q_{\mathrm{MC}}=0$. In contrast, the stable disk outside the instability strip is heated up and the temperature there increases because the extra heating term is added. On the other hand, the temperature decreases close to the inner edge in the models with a negative torque, i.e. when the energy is transferred from the disk to the black hole. The stable disk affected by the magnetic coupling is therefore cooled at the radii where $Q_{\mathrm{MC}}<0$ and heated at the region of $Q_{\mathrm{MC}}>0$.

Such a behavior of the temperature profile, which shows the net decrease of the temperature when the magnetic heating is added in the unstable region, is caused by the nature of the thermal/viscous instability. As discussed in detail in Janiuk et al. (2007), the instability is a result of a phase transition which occurs under certain conditions in the nuclear matter. As a result, the extra energy provided by the rotation of the black hole via the magnetic coupling is consumed by this phase transition and cannot heat the disk.

We conclude that the magnetic coupling between the spinning black hole and the disk does influence the thermal-viscous instability. It can result in shifting the unstable region towards a larger inner radius and shrinking it in size. The strength of the thermal-viscous instability will be modified under the influence of the magnetic coupling, because the density dip in the unstable region will be deeper. However, the temperature excess in the unstable region is smaller if the disk is magnetically coupled to the black hole. To quantify the net effect and calculate the resulting luminosity fluctuations we would need to make the timedependent modeling, which is beyond the scope of the present article. We intend to study this in more detail in the future work.

We also note that there is some small impact of magnetic coupling on the stable disk solutions. This is an extra dip in density accompanied with the temperature excess, which arises in the previously stable region. However, this effect seems to be 
much less pronounced than the thermal-viscous instability. This may potentially lead to the development of a separate type of a magnetic instability.

\section{Discussion}

The importance of the black hole spin in the accretion disk systems and the formation of jets, also in the presence of the magnetic fields, has been studied by many authors. It has been discussed e.g. in the review by Blandford (1999). In the standard accretion disk theory (Shakura \& Sunyaev 1973) it is assumed that there is no coupling between the black hole and the disk. However, if the magnetic field is considered, such a coupling may be present. Contrary to the Blandford-Znajek process (in which the magnetic filed lines threading the black hole may close on a very remote load, far away from the black hole), the magnetic field lines coupled to the accretion disk are closed in the vicinity of the inner radius. In this process, the energy and angular momentum are extracted from the black hole and transferred to the disk if the hole rotates faster than the disk (MacDonald \& Thorne 1982; Li 2000). Such coupling may have much higher efficiency in extracting the black hole rotation energy than the Blandford-Znajek mechanism, as was first quantitatively estimated by $\mathrm{Li}$ (2002).

Let us also remind here following $\mathrm{Li}$ (2002) that in this model the torque produced by the magnetic coupling with the black hole propagates only outwards, and no torque is imposed at the inner boundary of the disk. The non-zero torque at the inner boundary is possible if the disk is magnetically connected with the plasma in the transition region between the disk and the black hole (e.g. Krolik 1999), which we do not consider here. Another important assumption behind the present model is the one about the quasi steady-state of the disk and the magnetic field configuration. Under this assumption, the magnetic field lines are not tangled by the rotation, and MHD instabilities of the Balbus \& Hawley (1991) are not taken into account. In our present work we adopt this assumption, following $\mathrm{Li}$ (2002) and Wang et al. (2002, 2003a,b). The evolution of the coronal magnetic field threading the differentially rotating disk was studied in the literature, e.g. by Lovelace et al. (2002) in the context of Poynting flux dominated jets in quasars, and in Uzdensky (2004, 2005) by numerically solving the Grad-Shafranov equation in the Schwarzschild and Kerr metric.

In the context of gamma ray bursts, the black hole spin interaction with the magnetized torus was studied by van Putten et al. (2004). These authors take into account the dissipation of energy in the torus not only via the thermal and neutrino emission, but also through the gravitational waves and winds. They estimate the lifetime of the central engine powered by a rapidly spinning black hole, whose spin-down is governed by the surrounding magnetic field coupled to the inner torus. In the present model, we neglect the gravitational waves emission, and we concentrate on the structure of the neutrino-cooled disk. We restrict ourselves to the steady-state solution, but we note that the lifetime of the long gamma ray burst central engine is defined by the period of the rapid spin of the black hole, which may decrease with time during the engine activity (see also Janiuk et al. 2008).

We study the model of a stationary neutrino cooled disk around a rotating Kerr black hole. We confirmed the presence of the thermal-viscous instability in the region of the large neutrino opacity and efficient helium photodisintegration. The instability was first studied in Janiuk et al. (2007) in the time-dependent model for the Schwarzschild black hole. It was found to produce dramatic changes in the density and temperature profiles as well as a rapid time variability of the disk emission. Here, we quantitatively described the role of the black hole spin and viscosity in the disk. We found that in the Kerr black hole disks the large spin parameter results in a larger size of an unstable strip, which appears even for a moderate accretion rate. The critical accretion rate is anticorrelated with the $\mathrm{BH}$ spin required for the onset of the instability. In the extreme $\mathrm{BH}$ case, the instability close to the inner edge may be effective even for $\dot{M}=0.5 M_{\odot} \mathrm{s}^{-1}$. On the other hand, the more viscous the disk, the smaller in size is the unstable strip and the weaker should be the instability.

The time dependent calculations were not performed in the present work, because they would require to compute the time evolution of the $\mathrm{BH}$ spin and a moving radial grid, which is intended to be studied in the future work. However, we studied the influence of the black hole rotation on the disk structure considering our stationary model. This was done by means of the magnetic transfer of energy from the rotating black hole to the disk and vice versa.

The first important result in this work is that thermal instability occurs for quite low accretion rates, on the order of a fraction of solar mass per second, provided the black hole rotates very fast. This can be relevant for the origin of the variable energy input to the GRB jets not only in the case of the short GRBs, but also for long ones. The latter are presumably powered by the accretion of the fallback material from the massive star envelope onto the newly born black hole after the hypernova explosion (e.g. Mac Fadyen \& Woosley 1999). In these models, the accretion rate is found to be substantially lower than in the merger of two neutron stars, and therefore the event can last for a much longer time to power the GRB for several hundreds of seconds. On the other hand, the massive star that is a progenitor of a hypernova is a rotating star, and therefore a natural consequence of the explosion should be the rapidly rotating black hole in the center.

As was recently shown by Janiuk et al. (2008), the large black hole spin is a key ingredient for the occurrence of the longest duration GRBs, and is required for the GRB central engine to operate for a time on the order of hundreds of seconds. The present study leads us therefore to a general conclusion that the longest GRBs which are powered first by the neutrino annihilation and later by the black hole spin and require $a>0.9$, should be very variable at the initial phase of the prompt emission. Later on, when the accretion rate drops, the variability may be suppressed and what is observed is a smooth tail in the gamma ray lightcurve. On the other hand, the period of the highly variable GRB emission may be somewhat extended if the black hole is being spun up by the accretion. The issue discussed in the literature was whether in such a case the spin equilibrium value can be achieved at $a=0.998$ (Thorne 1974), or a less value (Gammie et al. 2004).

We also considered the heating of the innermost regions of the torus due to the magnetic coupling with the rotating black hole. We found that this process can have some influence on the thermal instability, because the size and position of the unstable region are somewhat changed. Also, the profiles of density and temperature in the unstable region are modified. The magnetic coupling enhances the change in the density profile, but it weakens the change in the temperature profile. The energy transfer to the disk from the rotating black hole does not suppress the thermal instability. This result also supports our conclusion that the variable and long duration GRBs are powered by the rapidly spinning black holes.

Acknowledgements. This work was partially supported by the Polish State Committee for Scientific Research grant NN 203512638 and the Polish 
A. Janiuk and Y.-F. Yuan: Unstable GRB disk around a spinning black hole

Astroparticle Network grant 621/E-78/SN-0068/2007. This work is also partially supported by National Basic Research Program of China (grant 2009CB824800), the National Natural Science Foundation (grant 10733010,10673010,10573016).

\section{References}

Balbus, S. A., \& Hawley, J. F. 1991, ApJ, 376, 214

Bardeen, J. M. 1970, Nature, 226, 64

Bardeen, J. M., Press, W. H., \& Teukolsky, S. A. 1972, ApJ, 178, 347

Beloborodov, A. M., Stern, B. E., \& Svensson, R. 2000, ApJ, 535, 158

Blandford, R. D. 1976, MNRAS, 176, 465

Blandford, R. D. 1999, in Astrophysical Discs - An EC Summer School, ed. J. A. Sellwood, \& J. Goodman, ASP Conf. Ser., 160, 265

[arXiv: astro-ph/9902001]

Blandford, R. D., \& Znajek, R. L. 1977, MNRAS, 179, 433

Chen, W. X., \& Beloborodov, A. M. 2007, ApJ, 657, 383

Cline, D., Czerny, B., Janiuk, A., Matthey, C., \& Otwinowski, S. 2005, ApJ, 663, L73

Di Matteo, T., Perna, R., \& Narayan, R. 2002, ApJ, 579, 706

Gammie, C. F., Shapiro, S. L., \& McKinney, J. C. 2004, ApJ, 602, 312

Janiuk, A., Perna, R., Di Matteo, T., \& Czerny, B. 2004, MNRAS, 355, 950

Janiuk, A., Yuan, Y. F., Di Matteo, T., \& Perna, R. 2007, ApJ, 664, 1011

Janiuk, A., Moderski, R., \& Proga, D. 2008, ApJ, 687, 433

Kohri, K., Narayan, R., \& Piran, T. 2005, 629, 341

Krolik, J. K. 1999, ApJ, 515, L73
Lei, W. H., Wang, D. X., Zhang, L., et al. 2009, ApJ, 700, 1970

Li, L.-X. 2000, ApJ, 533, L115

Li, L.-X. 2002, ApJ, 567, 463

Li, L.-X., \& Paczyński, B. 2000, ApJ, 534, L197

Lovelace, R. V. E., Li, H., Koldoba, A. V., Ustyugova, G. V., \& Romanova, M. 2002, ApJ, 572, 445

MacDonald, D., \& Thorne, K. S. 1982, MNRAS, 198, 345

Moderski, R., Sikora, M., \& Lasota, J.-P. 1998, MNRAS, 301, 142

Popham, R., \& Narayan, R. 1995, ApJ, 442, 337

Popham, R., Woosley, S. E., \& Fryer, C. 1999, ApJ, 518, 356

van Putten, M. H. P. M., Levinson, A., Lee, H. K., et al. 2004, Phys. Rev. D, 69, 044007

Reynoso, M. M., Romero, G. E., \& Sampayo, O. A. 2006, A\&A, 454, 11

Riffert, H., \& Herold, H. 1995, ApJ, 450, 508

Sawyer, R. F. 2003, Phys. Rev. D., 68, 6, 063001

Shakura, N. I., \& Sunyaev, R. A. 1973, A\&A, 24, 337

Thorne, K. S. 1974, ApJ, 191, 507

Uzdensky, D. A. 2004, ApJ, 603, 652

Uzdensky, D. A. 2005, ApJ, 620, 889

Wang, D. X., Xiao, K., \& Lei, W. H. 2002, MNRAS, 335, 655

Wang, D. X., Lei, W.-H., \& Ma, R.-Y. 2003a, MNRAS, 342, 851

Wang, D.-X., Ma, R.-Y., Lei, W.-H., \& Yao, G. Z. 2003b, ApJ, 595, 109

Yuan, Y. F. 2005, Phys. Rev. D, 72, 013007

Yuan, Y. F., \& Heyl, J. S. 2005, MNRAS, 360, 1493

Zhang, B. 2007, ChJAA, 7, 1 